\title{
Cambios en los hábitos alimentarios de los pacientes infectados por el VIH a través del asesoramiento gastronómico y talleres de adquisición de habilidades culinarias (Estudio ALíCIA-FLS)
}

\author{
Carla Estany Quera ${ }^{a, *}$, Anna Bonjoch ${ }^{a, b}$, Elena Roura ${ }^{c}$, Núria Cinca ${ }^{c}$ \\ a Fundación Lluita contra la Sida, Hospital Germans Trias i Pujol, Badalona, Barcelona, Spain. \\ b Unidad VIH, Hospital Germans Trias I Pujol, Badalona, Barcelona, Spain. \\ C Fundació Alícia, Sant Fruitós de Bages, Barcelona, Spain. \\ *cestany@flsida.org
}

Recibido el 13 de febrero de 2015; aceptado el 8 de mayo de 2015.

\section{CITA}

Estany Quera C, Bonjoch A, Roura E, Núria Cinca N. Cambios en los hábitos alimentarios de los pacientes infectados por el VIH a través del asesoramiento gastronómico y talleres de adquisición de habilidades culinarias (Estudio ALÍCIA-FLS). Rev Esp Nutr Hum Diet. 2015; 19(3): 184 - 186. DOI: 10.14306/renhyd.19.3.151

Aunque con la Terapia Antiretroviral de Gran Actividad (TARGA) han disminuido los casos de desnutrición y caquexia, persisten la disminución del apetito, ingesta alimentaria y pérdida de peso $^{1-7}$. La dietoterapia forma parte del tratamiento integral de personas con infección por VIH, dado el riesgo de sufrir trastornos nutricionales y posibles efectos adversos de las medicaciones que pueden tener un impacto negativo en el estado nutricional e ingesta alimentaria ${ }^{1-7}$. Varios estudios afirman que tener habilidades culinarias básicas, tanto pacientes como profesionales sanitarios, ayudan a comer mejor y a aumentar la adherencia a los mensajes y recomendaciones dietéticas, y que éstas son más eficaces si se acompañan de cursos o talleres de cocina ${ }^{8-9}$.

Se diseñó un estudio piloto observacional prospectivo, para evaluar si acompañar las pautas nutricionales estándar con talleres de cocina repercutía en el estado nutricional e ingesta alimentaria de pacientes infectados con el VIH con bajo peso. Se incluyeron 15 pacientes que se visitaban de forma rutinaria en la Unidad VIH del Hospital Germans Trias i Pujol (Badalona, Barcelona) y bajo peso (definido como IMC inferior a $20 \mathrm{~kg} / \mathrm{m}^{2}$ ). Se excluyeron pacientes con enfermedad grave concomitante, hepatitis descompensada, infección oportunista, enfermedad renal, uso de nutrición parenteral, uso de anabolizantes, tratamientos hormonales, orexígenos o estimulantes del apetito. El Comité Ético de nuestro centro aprobó el estudio y todos los individuos incluidos firmaron el consentimiento informado para su participación en el mismo.

Ambos grupos recibieron las mismas recomendaciones de dieta hipercalórica, hiperproteica y consejos de 
Tabla 1. Ingesta energética, macronutrientes y micronutrientes en grupo Control y grupo Talleres, en visita inicial y final. Datos expresados valor absoluto o porcentaje y mediana y rango intercuartílico.

\begin{tabular}{|c|c|c|c|c|c|}
\hline & \multicolumn{2}{|c|}{ Basal } & \multicolumn{2}{|c|}{ Final } & \multirow[b]{2}{*}{ p-valor } \\
\hline & Control & Talleres & Control & Talleres & \\
\hline Energía (kcal/día) & $2586,7(2266,3 ; 3002,1)$ & $2020,42(1719 ; 2448,1)$ & $2711,23(2154,8 ; 3507,9)$ & $2246,19(1720,4 ; 2578,1)$ & $p>0,05$ \\
\hline Proteína total (g) & $105,5(92,1 ; 124,2)$ & $93,1(86,9 ; 109,4)$ & $102,7(84,1 ; 127)$ & $94,935(76,2 ; 112,2)$ & $p>0,05$ \\
\hline Proteína \%VCT & $16,2(14,5 ; 18,9)$ & $17,9(14,7 ; 22,1)$ & $15,06(12,8 ; 17,6)$ & $16,95(15,8 ; 18,9)$ & $p>0,05$ \\
\hline Proteína vegetal (g) & $35,47(28 ; 39,8)$ & $25,06(21,1 ; 31,2)$ & $31,48(25,2 ; 36,5)$ & $28,015(21,5 ; 33,8)$ & $p>0,05$ \\
\hline Proteína animal (g) & $59,6(52,6 ; 83,6)$ & $60,4(42,3 ; 72)$ & $73,51(48,6 ; 74,7)$ & $60,64(45,5 ; 67,8)$ & $p>0,05$ \\
\hline Lípidos totales (g) & $112,56(84,6 ; 134,3)$ & $82,2(76,5 ; 101,9)$ & $136,26(80 ; 190,5)$ & $91,38(62,2 ; 112,8)$ & $p>0,05$ \\
\hline Lípidos \%VCT & $36,4(33,3 ; 42,5)$ & $37,1(34,5 ; 40,7)$ & $44,1(33,1 ; 49,1)$ & $35,7(32,1 ; 44)$ & $p>0,05$ \\
\hline AGS \%VCT & $9,8(8,9 ; 14,6)$ & $11,6(9,4 ; 13,2)$ & $12,6(7,4 ; 14,1)$ & $11,6(8,9 ; 12,3)$ & $p>0,05$ \\
\hline AGMI \%VCT & $17,3(15,8 ; 19,01)$ & $16,6(14 ; 19,8)$ & $19,2(14,6 ; 27,1)$ & $14,6(13,2 ; 19,9)$ & $p>0,05$ \\
\hline AGPI \%VCT & $6,4(4,2 ; 6,7)$ & $6,5(4,4 ; 7,6)$ & $7,5(4,9 ; 7,9)$ & $6,5(5,2 ; 7,5)$ & $p>0,05$ \\
\hline Colesterol (mg) & $229,5(191,7 ; 326,2)$ & $279,3(209,1 ; 365,2)$ & $408,4(318,1 ; 439,5)$ & $283,8(186,4 ; 371,5)$ & $p>0,05$ \\
\hline Glúcidos totales (g) & $308,2(246,6 ; 350,9)$ & $230(179,7 ; 296,4)$ & $287,2(263,5 ; 318,9)$ & $232,9(181,9 ; 281,4)$ & $p>0,05$ \\
\hline Glúcidos \%VCT & $47,7(40,4 ; 50,4)$ & $41,8(39,3 ; 48,6)$ & $40,0(36,5 ; 50,7)$ & $45,9(38,7 ; 48,9)$ & $p>0,05$ \\
\hline Azúcares digestibles (g) & $98,8(85,9 ; 141,7)$ & $76,1(61,1 ; 138,2)$ & $101,6(67,4 ; 145,5)$ & $94,0(63,3 ; 119,8)$ & $p>0,05$ \\
\hline Fibra total (g) & $35,5(20,2 ; 39,9)$ & $21,7(19,2 ; 35,6)$ & $22,0(20,1 ; 28,6)$ & $20,9(16,1 ; 25,4)$ & $p>0,05$ \\
\hline Etanol & $0,0(0 ; 0)$ & $0,2(0-4,3)$ & $0,0(0 ; 2,4)$ & $0,8(0 ; 4,5)$ & $p>0,05$ \\
\hline Sodio (mg) & $3062,7(2266,9 ; 3419)$ & $2458,7(2061,1 ; 2989,1)$ & $1901,6(1818,3 ; 3770,9)$ & $2310,8(1729 ; 2890,8)$ & $p>0,05$ \\
\hline Potasio (mg) & $4168,5(3146,8 ; 4572,7)$ & $3396,9(2557 ; 3975,9)$ & $3925,1(3261,5 ; 4515,4)$ & $3253,8(2644,2 ; 3953,3)$ & $p>0,05$ \\
\hline Calcio (mg) & $1135,5(820,4 ; 1494,9)$ & $1093,8(875,1 ; 1309,8)$ & $1194,9(569,1 ; 167,8)$ & $1024,9(538,9 ; 1364,5)$ & $p>0,05$ \\
\hline Magnesio (mg) & $357,2(285,3 ; 493,6)$ & $375,8(263,7 ; 421,6)$ & $\mathrm{Nd}$ & $0,81(0,73 ; 0,83)$ & $p>0,05$ \\
\hline Fósforo (mg) & $1652,8(1405,2 ; 1916,4)$ & $1481,9(1179,3 ; 1672,6)$ & $1584,7(1220,4 ; 2008,9)$ & $1336,6(1012,4 ; 1647,1)$ & $p>0,05$ \\
\hline Hierro (mg) & $13,5(11,5 ; 20,7)$ & $13,9(11,9 ; 18,3)$ & $16,1(13 ; 20,8)$ & $13,5(11,8 ; 14,5)$ & $p>0,05$ \\
\hline Zinc (mg) & $10,3(8,4 ; 16,9)$ & $10,0(9,2 ; 11,7)$ & $9,2(8,5 ; 18,4)$ & $9,9(8,8 ; 11,1)$ & $p>0,05$ \\
\hline Vitamina A total (mcg) & $1050,2(704,2 ; 2845,7)$ & $1035,6(539 ; 1594,2)$ & $783,6(589,4 ; 1439,6)$ & $594,1(365,3 ; 930,1)$ & $p>0,05$ \\
\hline Retinoides (mcg) & $215,4(158,2 ; 360,6)$ & $254,1(147,4 ; 320)$ & $369,5(160,8 ; 414,2)$ & $213,2(130,3 ; 284,8)$ & $p>0,05$ \\
\hline Carotenoides (mcg) & $4904,1(2721,9 ; 13681,1)$ & $2283,4(959,2 ; 6520,8)$ & $2190,9(1078,2 ; 5997,1)$ & $1751,6(669,3 ; 3129)$ & $p>0,05$ \\
\hline Vitamina D (mcg) & $2,3(1,1 ; 8,04)$ & $6,6(4,9 ; 10,2)$ & $8,7(1,7 ; 13,2)$ & $4,3(1,9 ; 16,1)$ & $p>0,05$ \\
\hline Vitamina E (mg e.t.) & $13,2(11,6 ; 17,04)$ & $12,3(9,4 ; 18,3)$ & $20,6(11,2 ; 22,2)$ & $10,1(8,5 ; 15,5)$ & $p>0,05$ \\
\hline Vitamina B1 (mg) & $1,6(1,3 ; 2,3)$ & $1,5(1,3 ; 2,5)$ & $2,2(1,6 ; 2,3)$ & $1,6(1,2 ; 2,1)$ & $p>0,05$ \\
\hline Vitamina B2 (mg) & $2,1(1,4 ; 2,7)$ & $1,8(1,5 ; 2,4)$ & $2,3(1,7 ; 3,1)$ & $1,4(1,3 ; 2,1)$ & $p>0,05$ \\
\hline Niacina (mg) & $23,9(21,7 ; 30,7)$ & $24,0(20 ; 29,9)$ & $27,8(20,3 ; 33)$ & $22,2(19,3 ; 27,9)$ & $p>0,05$ \\
\hline Vitamina B6 (mg) & $2,4(1,9 ; 3,3)$ & $2,4(2 ; 2,9)$ & $3,3(2,1 ; 3,6)$ & $2,5(1,9 ; 2,8)$ & $p>0,05$ \\
\hline Ácido fólico (mcg) & $345,0(259,8 ; 475,1)$ & $340,5(242,2 ; 372,1)$ & $418,9(319,2 ; 498,4)$ & $297,9(239,4 ; 355,4)$ & $p>0,05$ \\
\hline Vitamina B12 (mcg) & $6,6(3,2 ; 6,9)$ & $4,4(3,1 ; 7,5)$ & $5,5(3,8 ; 9,8)$ & $5,0(3,6 ; 6,6)$ & $p>0,05$ \\
\hline Vitamina C (mg) & $199,2(150,4 ; 217,5)$ & $116,7(93,8 ; 134,9)$ & $185,5(106,9)$ & $116,1(86,3 ; 160,3)$ & $p>0,05$ \\
\hline Ingesta calórica (kcal/kg/día) & 49,5 & 39,88 & 50,6 & 44,36 & $p>0,05$ \\
\hline Ingesta proteica (g/kg/día) & 1,85 & 1,96 & 1,92 & 1,86 & $p>0,05$ \\
\hline Requerimientos (kcal/día) & 2184,6 & 1879,9 & - & - & - \\
\hline Requerimientos (g proteínas/día) & 83,7 & 78 & - & - & - \\
\hline
\end{tabular}


enriquecimiento nutricional. El grupo Talleres recibió cuatro sesiones teórico-prácticas en la Fundación Alícia (Sant Fruitós de Bages, Barcelona), realizadas por un cocinero y una dietista-nutricionista expertos. Se trabajaron platos principales, así como estrategias culinarias para incrementar el valor proteico-energético de las preparaciones, de manera atractiva, sabrosa y adaptada a capacidades, habilidades culinarias y a situaciones de bajos recursos socio-económicos.

Ambos grupos aumentaron la ingesta oral, medida mediante Registro Dietético de 3 días consecutivos; en el grupo Talleres ésta fue superior. La ingesta de proteínas se mantuvo en ambos casos en valores superiores a las recomendaciones $(1,5 \mathrm{~g} / \mathrm{kg} / \mathrm{día})$ aunque se produjo una disminución respecto la basal en el grupo Talleres y un aumento en el grupo Control. La ingesta de grasas totales se mantuvo en porcentaje en el grupo Talleres y en el grupo Control la ingesta aumentó de forma franca en todos los parámetros. El aporte de fibra alimentaria disminuyó en ambos grupos. El resto de datos están descritos en la Tabla 1.

Como aspectos más destacables en la ingesta valorada mediante el Cuestionario de Frecuencia de Consumo de Alimentos, en el grupo Control aumentó la ingesta de alimentos ricos en grasa y azúcares simples (bebidas carbonatadas, mantequilla, bollería), alimentos ricos en proteínas como huevo, carne blanca, verduras y fruta desecada. En el grupo Talleres aumentaron cárnicos magros, fruta seca oleaginosa, verduras y hortalizas y algunos alimentos ricos en grasa y azúcares simples, aunque en menor medida que en el grupo control. En ambos grupos disminuyó la ingesta de fruta y pescado azul (fresco o en conserva).

Ambos grupos presentaron disminución de peso y de grasa en extremidades superiores medidos por Densitometria Dual de Rayos X (DEXA). En el grupo Control aumentó la grasa en tronco y extremidades inferiores. En el grupo Talleres aumentó la masa magra en todas las regiones excepto en piernas y tronco. La Densidad Mineral Osea (DMO) total aumentó en el grupo Control y disminuyó en el grupo Talleres; en ambos mejoró el T-score en región lumbar L1-L4 y empeoró en cuello de fémur. Dada la posible coexistencia de alteraciones de la distribución de grasa corporal en nuestros participantes, creemos que los cambios de peso y grasa son multifactoriales y el efecto de la dieta no se puede aislar en nuestro estudio.

La glucosa, albúmina, creatinina, PCR, colesterol total y colesterol LDL aumentaron y la prealbúmina disminuyó ambos grupos. El grupo Talleres presentó una mejora del perfil lipídico (no empeoramiento de las cifras de triglicéridos y aumento de colesterol $\mathrm{HDL}$ ).
Aunque no se haya observado un aumento de peso en los participantes, la ingesta de alimentos aumentó y se observaron mejoras en el perfil lipídico, que podrían justificar la recomendación de este tipo de actividades complementarias. Obviamente deben considerarse otras opciones de tratamiento del bajo peso o la caquexia. El presente estudio presenta varias limitaciones: el diseño de estudio piloto, la heterogenia y la inclusión pacientes con suplementación nutricional oral que presentan en escasa recuperación ponderal, aunque estas dos son características de nuestra población de estudio.

\section{$\longrightarrow$ CONFLICTO DE INTERESES}

$C E$, ER y NC declaran no tener ningún conflicto de interés. $A B$ ha recibido remuneraciones de ViiV, Jansen Cilag, Abbott y Roche.

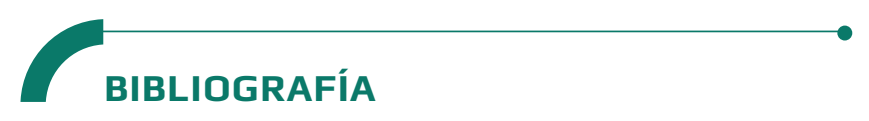

1. Mangili A, Murman DH, Zampini AM, Wanke CA. Nutrition and HIV infection: review of weight loss and wasting in the era of highly active antiretroviral therapy from the nutrition for healthy living cohort. Clin Infect Dis. 2006; 42(6): 836-42.

2. Grobler L, Siegfried N, Visser ME, Mahlungulu SS, Volmink J Nutritional interventions for reducing morbidity and mortality in people with HIV. Cochrane Database Syst Rev. 2013; 2: CD004536.

3. de Pee S, Semba RD. Role of nutrition in HIV infection: review of evidence for more effective programming in resource-limited settings. Food Nutr Bull. 2010; 31(4): S313-44.

4. Fields-Gardner C, Campa A; American Dietetics Association. Position of the American Dietetic Association: Nutrition Intervention and Human Immunodeficiency Virus Infection. ] Am Diet Assoc. 2010; 110(7): 1105-19.

5. Raiten DJ, Mulligan K, Papathakis P, Wanke C. Executive summary-nutritional care of HIV-infected adolescents and adults, including pregnant and lactating women: what do we know, what can we do, and where do we go from here? Am J Clin Nutr. 2011; 94(6): 1667S-1676S.

6. Leyes $P$, Martínez E, Forga Mde T. Use of diet, nutritional supplements and exercise in HIV-infected patients receiving combination antiretroviral therapies: a systematic review. Antivir Ther. 2008; 13(2): 149-59.

7. Chandrasekhar A, Gupta A. Nutrition and disease progression pre-highly active antiretroviral therapy (HAART) and postHAART: can good nutrition delay time to HAART and affect response to HAART? Am J Clin Nutr. 2011; 94(6): 1703S-1715S

8. Hartmann C, Dohle S, Siegrist M. Importance of cooking skills for balanced food choices. Appetite. 2013; 65: 125-31.

9. Chu YL, Farmer A, Fung C, Kuhle S, Storey KE, Veugelers PJ. Involvement in home meal preparation is associated with food preference and self-efficacy among Canadian children. Public Health Nutr. 2013; 16(1): 108-12. 